\title{
Robust Controllability and Observability Degrees of Polynomially Uncertain Systems
}

\author{
Somayeh Sojoudi ${ }^{\text {a }}$, Javad Lavaei ${ }^{\text {a }}$, Amir G. Aghdam ${ }^{\mathrm{b}}$ \\ ${ }^{a}$ Department of Control and Dynamical Systems, California Institute of Technology, Pasadena, CA 91125, USA \\ ${ }^{\mathrm{b}}$ Department of Electrical and Computer Engineering, Concordia University, Montréal, QC H3G 1M8, Canada
}

\begin{abstract}
This paper deals with the class of polynomially uncertain continuous-time linear time-invariant (LTI) systems whose uncertainties belong to a semi-algebraic set. The objective is to determine the minimum of the smallest singular value of the controllability or observability Gramian over the uncertainty region. This provides a quantitative measure for the robust controllability or observability degree of the system. To this end, it is shown that the problem can be recast as a sum-of-squares (SOS) problem. In the special case when the uncertainty region is polytopic, the corresponding SOS formulation can be simplified significantly. One can apply the proposed method to any large-scale interconnected system in order to identify those inputs and outputs that are more effective in controlling the system, in a robust manner. This enables the designer to simplify the control structure by ignoring those inputs and outputs whose contribution to the overall control operation is relatively weak. A numerical example is presented to demonstrate the efficacy of the results.
\end{abstract}

Key words: Analysis of systems with uncertainties, Optimization under uncertainties, Sum-of-squares, Large scale systems

\section{Introduction}

There has been a growing interest in recent years in robust control of systems with parametric uncertainty (Lavaei and Aghdam, 2008a; Oliveira and Peres, 2006; Chesi, Garulli, Tesi and Vicino, 2005; Oliveira and Geromel, 2005). The dynamic behavior of this type of systems is typically governed by a set of differential equations whose coefficients belong to fairly-known uncertainty regions. Although there are several methods to capture the uncertain nature of a real-world system (e.g., by modeling it as a structured or unstructured uncertainty (Dullerud and Paganini, 2005)), it turns out that the most realistic means of describing uncertainty is to parameterize it and then specify its domain of variation.

Robust stability is an important requirement in the control of a system with parametric uncertainty. This problem has been extensively studied in the case of linear

\footnotetext{
* This work has been supported by the Natural Sciences and Engineering Research Council of Canada (NSERC) under grant RGPIN-262127-07.

Email addresses: sojoudi@cds.caltech.edu (Somayeh Sojoudi), lavaei@cds.caltech.edu (Javad Lavaei), aghdam@ece.concordia.ca (Amir G. Aghdam).
}

time-invariant (LTI) control systems with specific types of uncertainty regions. for instance, sum-of-squares (SOS) relaxations are numerically efficient techniques introduced in Oliveira and Peres (2006) and Chesi, Garulli, Tesi and Vicino (2005) for checking the robust stability of polynomially uncertain systems. Moreover, a necessary and sufficient condition is proposed in Lavaei and Aghdam (2008a) for the robust stability verification of this class of uncertain systems, by solving a hierarchy of semi-definite programming (SDP) problems.

The concepts of controllability and observability were introduced in the literature, and it was shown that they play a key role in various feedback control analysis and design problems such as model reduction, optimal control, state estimation, etc. (Dullerud and Paganini, 2005). Several techniques are provided in the literature to verify the controllability and/or observability of a system. However, in many applications it is important to know how much controllable or observable a system is. Gramian matrices were introduced to address this issue by providing a quantitative measure for controllability and observability (Dullerud and Paganini, 2005). While these notions were originally introduced for fixed known systems, they have been investigated thoroughly in the past two decades for the case of uncertain systems (Savkin and Petersen, 1999; Ugrinovskii, 2005). 
On the other hand, real-world systems are often composed of multiple interacting components, and hence possess sophisticated structures. Such systems are typically modeled as large-scale interconnected systems, for which classical control analysis and design techniques are usually inefficient. Several results are reported in the literature for structurally constrained control of largescale systems in the contexts of decentralized and overlapping control, to address the shortcomings of the traditional control techniques (Davison and Chang, 1990; Siljak, 1991; Lavaei and Aghdam, 2007a, 2008b; Sojoudi and Aghdam, 2007).

This work aims to measure the minimum of the smallest singular value for the controllability and observability Gramians of parametric systems, over a given uncertainty region. Given a polynomially uncertain linear time-invariant (LTI) system with uncertain parameters defined on a semi-algebraic set, it is asserted that the controllability (observability) Gramian is a rational matrix in the corresponding parameters. It is desired to attain the minimum singular value of this matrix over the uncertainty region, but due to the rational structure of the matrix one cannot take advantage of the efficient techniques such as SOS tools. To bypass this obstacle, it is shown that said rational matrix can be replaced by a polynomial approximation which satisfies an important relation. An SOS formula is then obtained to find the underlying infimum. The special case of a polytopic uncertainty region is also investigated, due to its importance in practice. An alternative approach is proposed for this special case, with a substantially reduced computational burden.

The primary application of this work is to measure the robust closed-loop performance of a system subject to perturbation. Furthermore, the results obtained can be used in large-scale systems to determine the most important inputs and outputs in terms of robust performance.

This paper is organized as follows. The problem is formulated in Section 2, where some important background results are provided. The main results of the paper are developed in Section 3 for systems with polynomial uncertainty, and the special case of a polytopic region is also addressed in detail. The results are illustrated in Section 4 through a numerical example, and finally the concluding remarks are summarized in Section 5.

\section{Preliminaries and problem formulation}

Consider an uncertain LTI system $\mathcal{S}(\boldsymbol{\alpha})$ with the following state-space representation:

$$
\begin{aligned}
& \dot{x}(t)=A(\boldsymbol{\alpha}) x(t)+B(\boldsymbol{\alpha}) u(t) \\
& y(t)=C(\boldsymbol{\alpha}) x(t)+D(\boldsymbol{\alpha}) u(t)
\end{aligned}
$$

where
- $x(t) \in \Re^{n}$ is the state, and $u(t) \in \Re^{m}$ and $y(t) \in \Re^{r}$ are the input and output of the system, respectively.

- $\boldsymbol{\alpha}=\left[\alpha_{1}, \alpha_{2}, \ldots, \alpha_{k}\right]$ represents the uncertain parameters of the system, which are assumed to be fixed, but unknown. By assumption, this uncertainty vector belongs to a given semi-algebraic set $\mathcal{D}$ defined below:

$$
\mathcal{D}=\left\{\boldsymbol{\alpha} \in \Re^{k} \mid f_{1}(\boldsymbol{\alpha}) \geq 0, \ldots, f_{z}(\boldsymbol{\alpha}) \geq 0\right\}
$$

where $f_{1}(\boldsymbol{\alpha}), \ldots, f_{z}(\boldsymbol{\alpha})$ are given scalar polynomials.

- $A(\boldsymbol{\alpha}), B(\boldsymbol{\alpha}), C(\boldsymbol{\alpha})$ and $D(\boldsymbol{\alpha})$ are matrix polynomials in the variable $\boldsymbol{\alpha}$.

Assume that the matrix $A(\boldsymbol{\alpha})$ is robustly Hurwitz over the region $\mathcal{D}$ for all $\boldsymbol{\alpha} \in \mathcal{D}$ (this condition is required to define the infinite-horizon Gramians, and can be systematically checked using a variation of the SOS method proposed in Lavaei and Aghdam (2008a)). The controllability and observability of the system can be measured by the following parametric Gramian matrices, respectively:

$$
\begin{aligned}
& W_{c}(\boldsymbol{\alpha})=\int_{0}^{\infty} e^{A(\boldsymbol{\alpha}) t} B(\boldsymbol{\alpha}) B(\boldsymbol{\alpha})^{T} e^{A(\boldsymbol{\alpha})^{T} t} d t \\
& W_{o}(\boldsymbol{\alpha})=\int_{0}^{\infty} e^{A(\boldsymbol{\alpha})^{T} t} C(\boldsymbol{\alpha})^{T} C(\boldsymbol{\alpha}) e^{A(\boldsymbol{\alpha}) t} d t
\end{aligned}
$$

for all $\boldsymbol{\alpha} \in \mathcal{D}$. The matrices $W_{c}(\boldsymbol{\alpha})$ and $W_{o}(\boldsymbol{\alpha})$ can alternatively be obtained by solving the following continuoustime Lyapunov equations:

$$
\begin{aligned}
& A(\boldsymbol{\alpha}) W_{c}(\boldsymbol{\alpha})+W_{c}(\boldsymbol{\alpha}) A(\boldsymbol{\alpha})^{T}=-B(\boldsymbol{\alpha}) B(\boldsymbol{\alpha})^{T} \\
& A(\boldsymbol{\alpha})^{T} W_{o}(\boldsymbol{\alpha})+W_{o}(\boldsymbol{\alpha}) A(\boldsymbol{\alpha})=-C(\boldsymbol{\alpha})^{T} C(\boldsymbol{\alpha})
\end{aligned}
$$

Hence, one can write:

$$
\begin{gathered}
{[I \otimes A(\boldsymbol{\alpha})+A(\boldsymbol{\alpha}) \otimes I] \operatorname{vec}\left\{W_{c}(\boldsymbol{\alpha})\right\}} \\
=\operatorname{vec}\left\{-B(\boldsymbol{\alpha}) B(\boldsymbol{\alpha})^{T}\right\} \\
{\left[I \otimes A(\boldsymbol{\alpha})^{T}+A(\boldsymbol{\alpha})^{T} \otimes I\right] \operatorname{vec}\left\{W_{o}(\boldsymbol{\alpha})\right\}} \\
=\operatorname{vec}\left\{-C(\boldsymbol{\alpha})^{T} C(\boldsymbol{\alpha})\right\}
\end{gathered}
$$

where $\otimes$ denotes the Kronecker product, and $\operatorname{vec}\{\cdot\}$ is an operator which takes a matrix and converts it to a vector by stacking its columns on top of one another. It can be inferred from these equations that although $A(\boldsymbol{\alpha}), B(\boldsymbol{\alpha})$ and $C(\boldsymbol{\alpha})$ are polynomial matrices, the solutions $W_{c}(\boldsymbol{\alpha})$ and $W_{o}(\boldsymbol{\alpha})$ are symmetric rational matrices in $\boldsymbol{\alpha}$ (recall that the inverse of a polynomial matrix is a rational matrix).

Definition 1: The system $\mathcal{S}(\boldsymbol{\alpha})$ is said to be robustly controllable (observable), if it is controllable (observable) for every $\boldsymbol{\alpha} \in \mathcal{D}$.

It is well-known that the system $\mathcal{S}(\boldsymbol{\alpha})$ is robustly controllable (respectively, observable) if and only if $W_{c}(\boldsymbol{\alpha})$ 
(respectively, $\left.W_{o}(\boldsymbol{\alpha})\right)$ is positive definite for all $\boldsymbol{\alpha} \in$ $\mathcal{D}$ (Dullerud and Paganini, 2005). It follows from a celebrated result (Proposition 4.5 in Dullerud and Paganini (2005)) that the input energy required for controlling the system is, roughly speaking, proportional to the inverse of the matrix $W_{c}(\boldsymbol{\alpha})$, and more specifically, to the inverse of its smallest singular value. A similar result holds for the observability matrix (in a dual manner). This motivates the derivation of the minimum singular value of the matrices $W_{c}(\boldsymbol{\alpha})$ and $W_{o}(\boldsymbol{\alpha})$ over the region $\mathcal{D}$, which is central to this paper. Derivation of the minimum singular value will be addressed in the sequel for the matrix $W_{c}(\boldsymbol{\alpha})$ (evidently, the results developed for $W_{c}(\boldsymbol{\alpha})$ hold for the matrix $W_{o}(\boldsymbol{\alpha})$ as well $)$.

Notation 1: Given a matrix $M, \underline{\sigma}\{M\}$ denotes its minimum singular value.

Notation 2: Given a vector $\boldsymbol{\beta}=\left[\beta_{1}, \beta_{2}, \cdots, \beta_{k}\right]$, define $\boldsymbol{\beta}^{2}$ to be equal to $\boldsymbol{\beta}^{2}=\left[\beta_{1}^{2}, \beta_{2}^{2}, \cdots, \beta_{k}^{2}\right]$.

The following mild assumption on the region $\mathcal{D}$ is essential for the main development of this work.

Assumption 1 The set $\mathcal{D}$ is compact, and there exist $S O S$ scalar polynomials $w_{0}(\boldsymbol{\alpha}), w_{1}(\boldsymbol{\alpha}), \ldots, w_{z}(\boldsymbol{\alpha})$ such that all points $\boldsymbol{\alpha}$ satisfying the inequality:

$$
w_{0}(\boldsymbol{\alpha})+w_{1}(\boldsymbol{\alpha}) f_{1}(\boldsymbol{\alpha})+\cdots+w_{z}(\boldsymbol{\alpha}) f_{z}(\boldsymbol{\alpha}) \geq 0
$$

form a compact set.

There are two important points concerning Assumption 1. First, the validity of this assumption can be checked by solving a proper SOS problem. Furthermore, if the assumption does not hold, then the results of this paper will become only sufficient, as opposed to both necessary and sufficient.

\section{Main results}

As stated in the preceding section, the matrix $W_{c}(\boldsymbol{\alpha})$ satisfying the equality (4a) is a rational function, which impedes the use of the available SOS techniques. Since $W_{c}(\boldsymbol{\alpha})$ can be obtained from (3a), substituting the exponential matrices in the integral with their truncated Taylor series would result in a polynomial approximation of the Gramian matrix. However, the polynomial obtained using this simple technique would not necessarily satisfy an important property, namely inequality (11), which will be introduced later and is essential in developing the main results of this paper. This is due to the fact that the error of this truncation is sign-indefinite in general; i.e., it is neither positive definite nor negative definite. This obstacle will be overcome in the sequel.

One can adopt an approach similar to the one given in the proof of Lemma 1 in Lavaei and Aghdam (2008a) for discrete-time systems to conclude that $W_{c}(\boldsymbol{\alpha})$ can be expressed as $\frac{H(\boldsymbol{\alpha})}{h(\boldsymbol{\alpha})}$, where $H(\boldsymbol{\alpha})$ and $h(\boldsymbol{\alpha})$ are positive definite matrix and positive definite scalar polynomials (of known degrees), respectively, over the region $\mathcal{D}$. It follows from the positiveness of $h(\boldsymbol{\alpha})$ as well as the compactness of $\mathcal{D}$ that there exist reals $\mu_{1}$ and $\mu_{2}$ such that:

$$
0<\mu_{1}<h(\boldsymbol{\alpha})<\mu_{2}, \quad \forall \boldsymbol{\alpha} \in \mathcal{D}
$$

Definition 2: A sequence of matrices $\left\{M_{i}\right\}_{1}^{\infty}$ is said to converge to a matrix $M$ from below if $M_{1} \leq M_{2} \leq M_{3} \leq$ $\cdots \leq M$ and $\lim _{i \rightarrow \infty}\left\|M_{i}-M\right\|=0$.

Definition 3: Define $P_{i}(\boldsymbol{\alpha})$ to be:

$$
P_{i}(\boldsymbol{\alpha}):=W_{c}(\boldsymbol{\alpha}) \times\left(1-\left(1-\frac{h(\boldsymbol{\alpha})}{\mu_{2}}\right)^{2 i}\right), \quad i \in \mathbf{N}
$$

Theorem 1 The following statements are true:

i) $P_{i}(\boldsymbol{\alpha})$ is a matrix polynomial.

ii) Given $\boldsymbol{\alpha} \in \mathcal{D}$, the sequence $\left\{P_{i}(\boldsymbol{\alpha})\right\}_{1}^{\infty}$ converges to $W_{c}(\boldsymbol{\alpha})$ from below. In particular,

$$
\left(1-\left(1-\frac{\mu_{1}}{\mu_{2}}\right)^{2 i}\right) \min _{\boldsymbol{\alpha} \in \mathcal{D}} \underline{\sigma}\left\{W_{c}(\boldsymbol{\alpha})\right\} \leq \min _{\boldsymbol{\alpha} \in \mathcal{D}} \underline{\sigma}\left\{P_{i}(\boldsymbol{\alpha})\right\}
$$

and

$$
\min _{\boldsymbol{\alpha} \in \mathcal{D}} \underline{\sigma}\left\{P_{i}(\boldsymbol{\alpha})\right\} \leq \min _{\boldsymbol{\alpha} \in \mathcal{D}} \underline{\sigma}\left\{W_{c}(\boldsymbol{\alpha})\right\}
$$

for all $i \in \mathbf{N}$.

iii) $P_{i}(\boldsymbol{\alpha})$ satisfies the following matrix inequality:

$$
A(\boldsymbol{\alpha}) P_{i}(\boldsymbol{\alpha})+P_{i}(\boldsymbol{\alpha}) A(\boldsymbol{\alpha})^{T}+B(\boldsymbol{\alpha}) B(\boldsymbol{\alpha})^{T} \geq 0
$$

for all $\boldsymbol{\alpha} \in \mathcal{D}$.

Proof of Part (i): The proof of this part is an immediate consequence of the fact that the term:

$$
\left(1-\left(1-\frac{h(\boldsymbol{\alpha})}{\mu_{2}}\right)^{2 i}\right)
$$

is divisible by $h(\boldsymbol{\alpha})$ (the denominator of $W_{c}(\boldsymbol{\alpha})$ ).

Proof of Part (ii): It is straightforward to conclude from Definition 3 and the inequality (7) that:

$$
P_{1}(\boldsymbol{\alpha}) \leq P_{2}(\boldsymbol{\alpha}) \leq P_{3}(\boldsymbol{\alpha}) \leq \cdots
$$

and:

$$
P_{i}(\boldsymbol{\alpha}) \leq W_{c}(\boldsymbol{\alpha}) \leq\left(1-\left(1-\frac{\mu_{1}}{\mu_{2}}\right)^{2 i}\right)^{-1} P_{i}(\boldsymbol{\alpha})
$$


for all $\boldsymbol{\alpha} \in \mathcal{D}$. The proof of part (ii) follows directly from (13) and (14).

Proof of Part (iii): One can write:

$$
\begin{aligned}
& A(\boldsymbol{\alpha}) P_{i}(\boldsymbol{\alpha})+P_{i}(\boldsymbol{\alpha}) A(\boldsymbol{\alpha})^{T}+B(\boldsymbol{\alpha}) B(\boldsymbol{\alpha})^{T}= \\
& =B(\boldsymbol{\alpha}) B(\boldsymbol{\alpha})^{T}+\left(1-\left(1-\frac{h(\boldsymbol{\alpha})}{\mu_{2}}\right)^{2 i}\right) \\
& \times\left(A(\boldsymbol{\alpha}) W_{c}(\boldsymbol{\alpha})+W_{c}(\boldsymbol{\alpha}) A(\boldsymbol{\alpha})^{T}\right) \\
& =B(\boldsymbol{\alpha}) B(\boldsymbol{\alpha})^{T}\left(1-\frac{h(\boldsymbol{\alpha})}{\mu_{2}}\right)^{2 i} \geq 0
\end{aligned}
$$

This completes the proof.

Theorem 1 shows that $W_{c}(\boldsymbol{\alpha})$ can be approximated by a matrix polynomial satisfying a matrix inequality. Moreover, implicit bounds on the smallest singular value of the controllability matrix are provided. It is to be noted that as pointed out in Lavaei and Aghdam (2008a), one can use the an interpolation technique developed in Lavaei and Aghdam (2007b) to calculate $h(\boldsymbol{\alpha})$ (unlike $H(\boldsymbol{\alpha})$, whose calculation is involved). This makes it possible to obtain $\mu_{1}$ and $\mu_{2}$, and then use (9) in order to roughly determine a proper range of values for the degree of the polynomial which can approximate $W_{c}(\boldsymbol{\alpha})$ satisfactorily.

Remark 1 It can be verified that one candidate for the scalar polynomial $h(\boldsymbol{\alpha})$ is as follows:

$$
\begin{aligned}
h(\boldsymbol{\alpha}) & =(-1)^{n} \operatorname{det}(I \otimes A(\boldsymbol{\alpha})+A(\boldsymbol{\alpha}) \otimes I) \\
& =(-1)^{n} \prod_{i=1}^{n} \prod_{j=1}^{n}\left(\lambda_{i}(\boldsymbol{\alpha})+\lambda_{j}(\boldsymbol{\alpha})\right)
\end{aligned}
$$

where $\lambda_{1}(\boldsymbol{\alpha}), \ldots, \lambda_{n}(\boldsymbol{\alpha})$ denote the eigenvalues of the matrix $A(\boldsymbol{\alpha})$. As a result, one can easily relate $\mu_{1}$ and $\mu_{2}$ to the minimum and maximum magnitude of the eigenvalues of $A(\boldsymbol{\alpha})$ over the region $\mathcal{D}$ (roughly speaking). Therefore, the ratio $\frac{\mu_{1}}{\mu_{2}}$ quantifies the degree of uncertainty of the open-loop system (i.e. the matrix $A(\boldsymbol{\alpha})$ ) in terms of the location of the eigenvalues.

Let an optimization problem be introduced in the sequel.

Optimization 1 Given the system $\mathcal{S}(\boldsymbol{\alpha})$ and the uncertainty region $\mathcal{D}$, maximize the real-valued scalar variable $\mu$ subject to the constraint that there exist a symmetric matrix polynomial $P(\boldsymbol{\alpha})$ and SOS matrix polynomials
$S_{0}(\boldsymbol{\alpha}), \ldots, S_{z}(\boldsymbol{\alpha}), \tilde{S}_{0}(\boldsymbol{\alpha}), \ldots, \tilde{S}_{z}(\boldsymbol{\alpha})$ such that:

$$
\begin{aligned}
& A(\boldsymbol{\alpha}) P(\boldsymbol{\alpha})+P(\boldsymbol{\alpha}) A(\boldsymbol{\alpha})^{T}+\tilde{B}(\boldsymbol{\alpha}) \tilde{B}(\boldsymbol{\alpha})^{T}= \\
& =S_{0}(\boldsymbol{\alpha})+\sum_{i=1}^{z} S_{i}(\boldsymbol{\alpha}) f_{i}(\boldsymbol{\alpha}) \\
& P(\boldsymbol{\alpha})=\mu I_{n}+\tilde{S}_{0}(\boldsymbol{\alpha})+\sum_{i=1}^{z} \tilde{S}_{i}(\boldsymbol{\alpha}) f_{i}(\boldsymbol{\alpha})
\end{aligned}
$$

where $I_{n}$ is the $n \times n$ identity matrix. Denote the solution of this optimization problem with $\mu^{*}$.

Theorem 2 The quantity $\min _{\boldsymbol{\alpha} \in \mathcal{D}} \underline{\sigma}\left\{W_{c}(\boldsymbol{\alpha})\right\}$ is equal to $\mu^{*}$.

Proof: The proof is based on Theorem 1, Assumption 1 and the results of Scherer and Hol (2006) (Theorem 2). The details can be found in Sojoudi, Lavaei and Aghdam (2009).

Remark 2 It can be observed that Optimization 1 is an SOS optimization problem, which can be efficiently handled using proper software such as YALMIP or SOSTOOLS (Lofberg, 2004; Prajna, Papachristodoulou, Seiler and Parrilo, 2004). Nevertheless, it is first required to consider some upper bounds a priori on the degrees of the polynomials involved in the corresponding optimization problem, from which a lower bound on the solution of Optimization 1 can be found. In other words, this optimization problem can be formulated as a hierarchy of SDP problems, whose solutions converge asymptotically to the quantity of interest, i.e. $\min _{\boldsymbol{\alpha} \in \mathcal{D}} \underline{\sigma}\left\{W_{c}(\boldsymbol{\alpha})\right\}$, from below.

\subsection{Special case: A polytopic region}

Although Theorem 2 provides a numerically tractable method for measuring the robust controllability of a system, the proposed optimization problem can be simplified significantly for special cases of interest. For instance, assume that $\mathcal{D}$ is a polytopic region $\mathcal{P}$ given by:

$$
\mathcal{P}=\left\{\boldsymbol{\alpha} \mid \alpha_{1}+\cdots+\alpha_{k}=1, \alpha_{1}, \ldots, \alpha_{k} \geq 0\right\}
$$

This type of uncertainty region is of particular interest, due to its important applications.

Assumption 2 Assume that $A(\boldsymbol{\alpha})$ and $\tilde{B}(\boldsymbol{\alpha})$ are homogeneous matrix polynomials, and let their degrees be denoted by $\zeta_{1}$ and $\zeta_{2}$, respectively.

Note that Assumption 2 holds automatically for polytopic systems, with $\zeta_{1}=\zeta_{2}=1$.

Theorem 3 The quantity $\min _{\boldsymbol{\alpha} \in \mathcal{P}} \underline{\sigma} W_{c}(\boldsymbol{\alpha})$ is equal to the maximum value of $\mu$ for which there exists a homogeneous matrix polynomial $\tilde{P}(\boldsymbol{\alpha})$ satisfying the inequalities 


$$
\begin{aligned}
& \tilde{P}\left(\boldsymbol{\alpha}^{2}\right) \geq 0 \\
& {\left[A\left(\boldsymbol{\alpha}^{2}\right)\left(\tilde{P}\left(\boldsymbol{\alpha}^{2}\right)+\mu\left(\boldsymbol{\alpha} \boldsymbol{\alpha}^{T}\right)^{\zeta_{3}} I_{n}\right)+\left(\tilde{P}\left(\boldsymbol{\alpha}^{2}\right)+\mu\left(\boldsymbol{\alpha} \boldsymbol{\alpha}^{T}\right)^{\zeta_{3}} I_{n}\right) A^{T}\left(\boldsymbol{\alpha}^{2}\right)\right]\left(\boldsymbol{\alpha} \boldsymbol{\alpha}^{T}\right)^{\max \left(0,2 \zeta_{2}-\zeta_{1}-\zeta_{3}\right)}} \\
& +B\left(\boldsymbol{\alpha}^{2}\right) B\left(\boldsymbol{\alpha}^{2}\right)^{T}\left(\boldsymbol{\alpha} \boldsymbol{\alpha}^{T}\right)^{\max \left(0, \zeta_{3}+\zeta_{1}-2 \zeta_{2}\right)} \geq 0
\end{aligned}
$$

given in (19) for all $\boldsymbol{\alpha} \in \mathbf{R}^{k}$, where $\zeta_{3}$ denotes the degree of the polynomial $\tilde{P}(\boldsymbol{\alpha})$.

Proof: For a complete proof of this theorem, one can refer to Sojoudi, Lavaei and Aghdam (2009).

Optimization 2 Maximize $\mu$ subject to the constraint that there exist a homogeneous matrix polynomial $P(\boldsymbol{\alpha})$ and $S O S$ matrix polynomials $S_{1}(\boldsymbol{\alpha})$ and $S_{2}(\boldsymbol{\alpha})$ such that:

$$
\begin{aligned}
& P\left(\boldsymbol{\alpha}^{2}\right)=S_{1}(\boldsymbol{\alpha}) \\
& {\left[A\left(\boldsymbol{\alpha}^{2}\right)\left(P\left(\boldsymbol{\alpha}^{2}\right)+\mu\left(\boldsymbol{\alpha} \boldsymbol{\alpha}^{T}\right)^{\zeta_{3}} I_{n}\right)\right.} \\
& \left.+\left(P\left(\boldsymbol{\alpha}^{2}\right)+\mu\left(\boldsymbol{\alpha} \boldsymbol{\alpha}^{T}\right)^{\zeta_{3}} I_{n}\right) A^{T}\left(\boldsymbol{\alpha}^{2}\right)\right] \\
& \times\left(\boldsymbol{\alpha} \boldsymbol{\alpha}^{T}\right)^{\max \left(0,2 \zeta_{2}-\zeta_{1}-\zeta_{3}\right)} \\
& +\tilde{B}\left(\boldsymbol{\alpha}^{2}\right) \tilde{B}\left(\boldsymbol{\alpha}^{2}\right)^{T}\left(\boldsymbol{\alpha} \boldsymbol{\alpha}^{T}\right)^{\max \left(0, \zeta_{3}+\zeta_{1}-2 \zeta_{2}\right)}=S_{2}(\boldsymbol{\alpha})
\end{aligned}
$$

where $\zeta_{3}$ denotes the degree of the polynomial $P(\boldsymbol{\alpha})$. Denote the solution of this optimization problem with $\tilde{\mu}^{*}$.

The following lemma is required in order to delve into the properties of the optimal parameter $\tilde{\mu}^{*}$ defined above.

Lemma 1 Let $M(\boldsymbol{\alpha})$ be a homogeneous matrix polynomial with the property that $M\left(\boldsymbol{\alpha}^{2}\right)$ is positive definite for every $\boldsymbol{\alpha} \in \mathbf{R}^{k} \backslash\{0\}$. There exists a natural number $c$ so that $\left(\boldsymbol{\alpha} \boldsymbol{\alpha}^{T}\right)^{c} M\left(\boldsymbol{\alpha}^{2}\right)$ is $S O S$.

Proof: The proof of this lemma relies heavily on the extension of Polya's theorem ( Hardy, Littlewood and Polya, 1952) to the matrix case, as carried out in Scherer and Hol (2006). More precisely, since $M(\boldsymbol{\alpha})$ is positive definite over the polytope $\mathcal{P}$, it follows from Theorem 3 in Scherer and Hol (2006) that there exists a natural number $c$ such that $\left(\alpha_{1}+\alpha_{2}+\cdots+\alpha_{k}\right)^{c} M(\boldsymbol{\alpha})$ has only positive-semidefinite matrix coefficients. This implies that the coefficients of $\left(\boldsymbol{\alpha} \boldsymbol{\alpha}^{T}\right)^{c} M\left(\boldsymbol{\alpha}^{2}\right)$ are all positive-semidefinite, and in addition, its monomials are squared terms. As a result, $\left(\boldsymbol{\alpha} \boldsymbol{\alpha}^{T}\right)^{c} M\left(\boldsymbol{\alpha}^{2}\right)$ is SOS.

Theorem 4 The quantity $\min _{\boldsymbol{\alpha} \in \mathcal{P}}\left\{W_{c}(\boldsymbol{\alpha})\right\}$ is equal to $\tilde{\mu}^{*}$.

Proof: The proof will be performed in two steps. First, observe that if (20) holds for some matrices $P(\boldsymbol{\alpha}), S_{1}(\boldsymbol{\alpha})$ and $S_{2}(\boldsymbol{\alpha})$, then (19) is satisfied for $\tilde{P}(\boldsymbol{\alpha})=P(\boldsymbol{\alpha})$.
Conversely, assume that a matrix polynomial $\tilde{P}(\boldsymbol{\alpha})$ satisfies the inequalities given in (19), with the non-strict inequalities replaced by strict inequalities for every $\boldsymbol{\alpha} \in$ $\mathbf{R}^{k} \backslash\{0\}$ (such strict inequalities correspond to the case when the term maximum is substituted by supremum). Note that for a strict inequality in (19a), one would need to replace $\tilde{P}(\boldsymbol{\alpha})$ and $\mu$ with $\tilde{P}(\boldsymbol{\alpha})-\varepsilon\left(\boldsymbol{\alpha} \boldsymbol{\alpha}^{T}\right)^{\zeta_{3}}$ and $\mu+\varepsilon$, respectively, for a positive infinitesimal number $\varepsilon$. Now, one can apply Lemma 1 to these inequalities to conclude that there exists a natural number $c$ such that if the expressions in the left sides of the inequalities (19a) and $(19 b)$ are multiplied by $\left(\boldsymbol{\alpha} \boldsymbol{\alpha}^{T}\right)^{c}$, then they become SOS matrix polynomials. It is enough to choose $P(\boldsymbol{\alpha})$ as $\tilde{P}(\boldsymbol{\alpha})\left(\boldsymbol{\alpha} \boldsymbol{\alpha}^{T}\right)^{c}$ for the inequalities given in (20) to hold (for some appropriate matrices $S_{1}(\boldsymbol{\alpha})$ and $S_{2}(\boldsymbol{\alpha})$ ).

Corollary 1 The solution of Optimization 2 is a monotone nondecreasing function with respect to $\zeta_{3}$ (the degree of the polynomial $\tilde{P}(\boldsymbol{\alpha})$ ).

Proof: The proof is a direct consequence of the fact that if $\tilde{P}(\boldsymbol{\alpha})$ satisfies the constraints of Optimization 2 for some $\mu$, then $\tilde{P}(\boldsymbol{\alpha})\left(\alpha_{1}+\cdots+\alpha_{k}\right)$ also satisfies them for the same $\mu$, but for some other suitable matrices $S_{1}(\boldsymbol{\alpha})$ and $S_{2}(\boldsymbol{\alpha})$.

As far as the complexity is concerned, it is easy to verify that Optimization 2 introduced in the present work is basically as complex as the optimization problem tackled in Chesi, Garulli, Tesi and Vicino (2005). This partly results from the fact that there are an SOS homogeneous polynomial and two SOS constraints of a particular form in both approaches. For a detailed comparison between the complexities of the results given in Chesi, Garulli, Tesi and Vicino (2005), Oliveira and Peres (2006) and Lavaei and Aghdam (2008a), the interested reader may refer to Lavaei and Aghdam (2008a).

\subsection{Application to large-scale systems}

In large-scale interconnected systems, typically the input and output vectors $u(t)$ and $y(t)$ have several entries. Due to the practical limitations, in this type of systems it is desired to simplify the control structure and employ as few communication links as possible (a communication link in an interconnected system is referred to a data transmission channel between a pair of local controllers). In other words, it would be very useful to extract two subvectors $\tilde{u}(t)$ and $\tilde{y}(t)$ from the vectors $u(t)$ and $y(t)$ such that the system is controllable from the 
reduced input $\tilde{u}(t)$ and observable from the reduced output $\tilde{y}(t)$. Moreover, the controllability and observability of the new configuration must be sufficiently strong. To find the proper subvectors $\tilde{u}(t)$ and $\tilde{y}(t)$, one can consider all desirable subsets of the inputs and outputs, and calculate the minimum singular values of the Gramians for each combination to assess the corresponding controllability and observability degrees. One can then choose the optimal subset of the input and output vectors, accordingly. This idea is further clarified in the next section.

\section{Numerical example}

Example 1 Consider an uncertain fourth-order LTI system with the matrices $A\left(\alpha_{1}, \alpha_{2}\right)$ and $B\left(\alpha_{1}, \alpha_{2}\right)$ given in the numerical example of Sojoudi, Lavaei and Aghdam (2009), where $\alpha_{1}$ and $\alpha_{2}$ are the uncertain parameters of the system, which belong to the polytope $\mathcal{P}=\left\{\left(\alpha_{1}, \alpha_{2}\right) \mid \alpha_{1}+\alpha_{2}=1, \alpha_{1}, \alpha_{2} \geq 0\right\}$. Regard this system as an interconnected system with four inputs. It is desired to determine which inputs contribute weakly to the control of the system, and hence can be ignored for the sake of cost reduction. In other words, the objective is to find out which inputs play a vital role in controlling the system. To this end, for any given set $g \subset\{1,2,3,4\}$ let $\mathcal{S}^{g}(\boldsymbol{\alpha})$ represent the system $\mathcal{S}(\boldsymbol{\alpha})$ after ignoring those inputs whose indices belong to $g$. Denote the controllability Gramian of this system with $W_{c}^{g}(\boldsymbol{\alpha})$.

Given $\boldsymbol{\alpha} \in \mathcal{P}$ and a final state $x_{0}$ of unit norm, consider the problem of finding an input $u(t)$ over the time interval $(-\infty, 0]$ with minimum $L_{2}$ norm such that it drives the state of the system $\mathcal{S}^{g}(\boldsymbol{\alpha})$ from $x(-\infty)=0$ to $x(0)=x_{0}$. As discussed in Section 4.3 of Dullerud and Paganini (2005), one possible solution is given by:

$$
u_{o p t}(t)=B^{g}(\boldsymbol{\alpha})^{T} e^{-A(\boldsymbol{\alpha})^{T} t} W_{c}^{g}(\boldsymbol{\alpha})^{-1} x_{0}, \quad t \leq 0
$$

where $B^{g}(\boldsymbol{\alpha})$ is the $B$-matrix of the system $\mathcal{S}^{g}(\boldsymbol{\alpha})$. The optimal input energy can be computed as:

$$
\left\|u_{\text {opot }}\right\|^{2}=x_{0}^{T} W_{c}^{g}(\boldsymbol{\alpha})^{-1} x_{0}
$$

Define $\nu(g)$ to be the maximum value of this optimal input energy over all final states $x_{0}$ of unit norm and all $\boldsymbol{\alpha} \in \mathcal{P}$. The idea behind this definition is that $\nu(g)$ provides an upper bound for the input energy required to drive the state of the uncertain system $\mathcal{S}^{g}(\boldsymbol{\alpha}), \boldsymbol{\alpha} \in \mathcal{P}$, from the origin at time $t=-\infty$ to any arbitrary point in the unit ball at time $t=0$. Note that if $\nu(g)$ corresponds to a final state $x_{0}^{*}$ and an uncertain parameter $\boldsymbol{\alpha}^{*}$, then $x_{0}^{*}$ must be a unit eigenvector of $W_{c}^{g}\left(\boldsymbol{\alpha}^{*}\right)$ associated with its smallest eigenvalue (singular value). This relationship can be expressed by:

$$
\frac{1}{\nu(g)}=\min _{\alpha \in \mathcal{P}} \underline{\sigma}\left\{W_{c}^{g}(\boldsymbol{\alpha})\right\}
$$

The objective is to evaluate $\nu(g)$ for different choices of $g$. To this end, four cases are considered as follows:

- Case 1: $g=\{2,3\}$.

- Case 2: $g=\{1\}$.

- Case 3: $g=\{4\}$.

- Case $4: g=\{\}$.

To obtain $\nu(g)$ for any of the above cases, it suffices to solve Optimization 2 with the appropriate matrix $B^{g}(\boldsymbol{\alpha})$. For this purpose, the order of the polynomial $P(\boldsymbol{\alpha})$ being sought should be chosen a priori. This optimization is treated using YALMIP on a Dell laptop with a $1.6 \mathrm{GHz}$ processor and $512 \mathrm{MB}$ memory, and the results are given in Table 1 . The last column of this table gives the points $\left(\alpha_{1}^{*}, \alpha_{2}^{*}\right)$ for which the largest optimal input energy $\nu(g)$ is required. These points are computed by gridding the polytope properly, and performing an exhaustive search. Therefore, the entries of the last column are computed using a brute force technique, which will be exploited to verify the results obtained by solving Optimization 2. The second column of the table gives the solution of Optimization 2 at the second relaxation, i.e., when $P(\boldsymbol{\alpha})$ is assumed to be a homogeneous polynomial of order 2 (with the monomials $\alpha_{1}^{2}, \alpha_{2}^{2}, \alpha_{1} \alpha_{2}$ ). It can be verified that the solution obtained for any of the cases 1 , 2 or 3 corresponds to the minimum singular value of the Gramian matrix evaluated at the optimal point given in the last column of the table. This proves that the relaxation arrives at the correct solution for cases 1,2 , and 3 . For case 4, Optimization 2 is also solved at the fourth relaxation (by considering the monomials $\alpha_{1}^{4}, \alpha_{1}^{3} \alpha_{2}, \alpha_{1}^{2} \alpha_{2}^{2}$, $\alpha_{1}^{1} \alpha_{2}^{3}, \alpha_{2}^{4}$ for $\left.P(\boldsymbol{\alpha})\right)$. The corresponding solution is given in the third column, which is, in fact, the exact optimal value. The CPU time consumed for solving Optimization 2 at the second relaxation is given in the fourth column for each case; these values show that the problem is solved very fast. Using the results in columns 2 and 3 of the table as well as the equation (23), the quantity $\nu(g)$ is calculated and provided in column 5 . It is worth mentioning that the ratio $\frac{\mu_{1}}{\mu_{2}}$ is equal to 0.075 in this example.

The values given in Table 1 (case 2 ) imply that the first input of the system is fairly important and ignoring it in the controller design would substantially increase the control energy required for shifting the state vector from certain points in the state-space. In contrast, the last input may be neglected, because its contribution is not significant as reflected by the small minimum singular value (case 3). However, if the second and third inputs are ignored concurrently (case 1), although the system remains robustly controllable by the remaining inputs, the required control energy would be huge.

For each of the above-mentioned cases, let the optimal input corresponding to the worst-case scenario (i.e. the final state $x_{0}^{*}$ and the uncertain variable $\boldsymbol{\alpha}^{*}$ ) be applied 
Table 1

Numerical results for Example 1

\begin{tabular}{|c|c|c|c|l|c|}
\hline Case & $\begin{array}{c}\text { Second } \\
\text { relaxation }\end{array}$ & $\begin{array}{c}\text { Fourth } \\
\text { relaxation }\end{array}$ & $\begin{array}{c}\text { CPU time for } \\
\text { second relaxation }\end{array}$ & $\nu(g)$ & $\left(\alpha_{1}^{*}, \alpha_{2}^{*}\right)$ \\
\hline 1 & 0.0068 & $*$ & $0.88 \mathrm{sec}$ & 147.06 & $(0.820,0.180)$ \\
\hline 2 & 0.0744 & $*$ & $0.82 \mathrm{sec}$ & 13.44 & $(0.240,0.760)$ \\
\hline 3 & 0.1920 & $*$ & $0.78 \mathrm{sec}$ & 5.21 & $(0.225,0.775)$ \\
\hline 4 & 0.2516 & 0.2531 & $0.85 \mathrm{sec}$ & 3.97 & $(0.234,0.766)$ \\
\hline
\end{tabular}
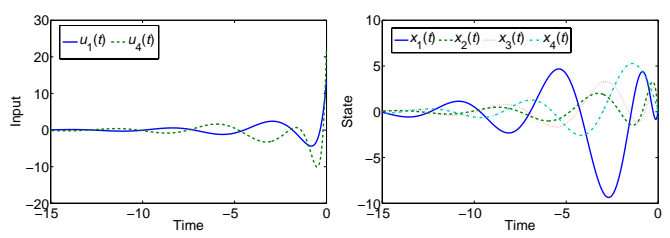

Fig. 1. The input and state of the system in case 1 (i.e., when inputs 2 and 3 are blocked).
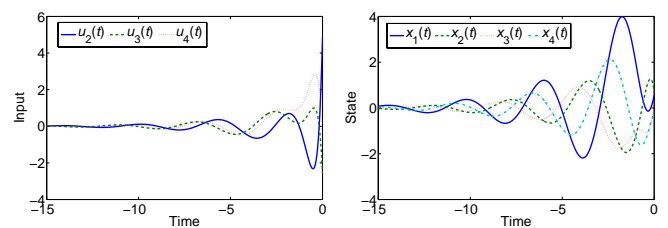

Fig. 2. The input and state of the system in case 2 (i.e., when input 1 is blocked).
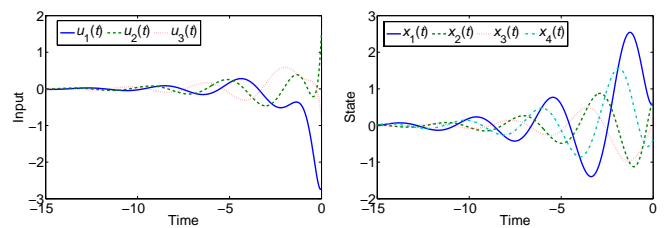

Fig. 3. The input and state of the system in case 3 (i.e., when input 4 is blocked).

to the system. Note that this input is given by (21), with $\boldsymbol{\alpha}=\boldsymbol{\alpha}^{*}$ (provided in Table 1) and $x_{0}=x_{0}^{*}$ as defined earlier. The resulting input and state of the system are plotted in Figures 1, 2, 3, and 4. Notice that although these signals extend from $t=-\infty$ to $t=0$, they are sketched only on the interval $-15 \leq t \leq 0$. These figures confirm the theoretical results obtained in this work. For instance, one can observe that in the case when both inputs $u_{2}(t)$ and $u_{3}(t)$ are blocked, the worst-case optimal input of the system has a large overshoot occurring at $t=0$ (about 22 in magnitude).

\section{Conclusions}

Given a continuous-time linear time-invariant (LTI) system which is polynomially uncertain on a semi-algebraic
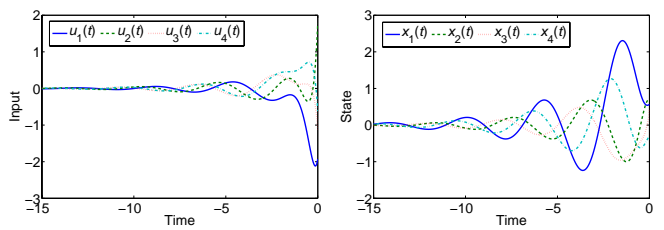

Fig. 4. The input and state of the system in (i.e., when none of the inputs is blocked).

region, this paper obtains the minimum of the smallest singular value of its controllability (observability) Gramian matrix. For this purpose, it is first shown that the Gramian is a rational function which can be approximated by a matrix polynomial (with any arbitrary precision) that satisfies an important relation. A sumof-squares (SOS) formula is then derived for solving the underlying problem, which can be efficiently handled using proper software. An alternative SOS method is subsequently obtained for the case when the uncertainty region is a polytope. This allows one to measure the robust controllability (observability) degree of the system, when the parameters of the system are subject to perturbation. The method proposed here can be used to find a dominant subset of inputs and outputs for any given large-scale system, by determining the effectiveness of each input and output in the overall operation of the control system. Simulations demonstrate the effectiveness of the proposed results.

\section{References}

Bliman, P. A., Oliveira, R. C. L. F., Montagner, V. F., \& Peres, P. L. D. (2006). Existence of homogeneous polynomial solutions for parameter-dependent linear matrix inequalities with parameters in the simplex. Proc. 45th IEEE Conf. Decision and Contr. San Diego, CA (pp. 1486-1491)

Chesi, G. (2008). On the non-conservatism of a novel LMI relaxation for robust analysis of polytopic systems. Automatica. 44(11), 2973-2976.

Chesi, G., Garulli, A., Tesi, A., \& Vicino, A. (2005). Polynomially parameter-dependent Lyapunov functions for robust stability of polytopic systems: an LMI approach. IEEE Trans. Automat. Contr. 50(3), 365-370. 
Davison, E. J., \& Chang, T. N. (1990). Decentralized stabilization and pole assignment for general proper systems. IEEE Trans. Automat. Contr. 35(6), 652664.

Dullerud, G. E., \& Paganini, F. (2005). A course in robust control theory: A convex approach. Texts in Applied Mathematics, Springer.

Hardy, G. H., Littlewood, J. E., \& Polya, G. (1952). Inequalities. Cambridge University Press, Cambridge, UK, Second edition.

Hillar, C. J., \& Nie, J. (2008). An elementary and constructive solution to Hilbert's 17th Problem for matrices. Proc. American Mathematical Society. 136, 73-76.

Lavaei, J., \& Aghdam, A. G. (2007a). A graph theoretic method to find decentralized fixed modes of LTI systems. Automatica. 43(12), 2129-2133.

Lavaei, J., \& Aghdam, A. G. (2007b). Optimal periodic feedback design for continuous-time LTI systems with constrained control structure. International Journal of Control. 80(2), 220-230.

Lavaei, J., \& Aghdam, A. G. (2008a). Robust stability of LTI systems over semi-algebraic sets using sum-ofsquares matrix polynomials. IEEE Trans. Automat. Contr. 53(1), 417-423.

Lavaei, J., \& Aghdam, A. G. (2008b). Control of continuous-time LTI systems by means of structurally constrained controllers. Automatica. 44(1), 141-148.

Sojoudi, S., Lavaei, J., \& Aghdam, A. G. (2009). Robust controllability and observability degrees of polynomially uncertain systems. To appear in Proceedings of 48 th IEEE Conference on Decision and Control, Shanghai, China.

Lofberg, J. (2004). A toolbox for modeling and optimization in MATLAB. Proc. of the CACSD Conf. Taipei, Taiwan (available online at http://control.ee.ethz.ch/ joloef/yalmip.php).

Oliveira, M. C. de, \& Geromel, J. C. (2005). A class of robust stability conditions where linear parameter dependence of the Lyapunov function is a necessary condition for arbitrary parameter dependence. Sys. Contr. Lett. 54(11), 1131-1134.

Oliveira, R. C. L. F., Oliveira, M. C. de, \& Peres, P. L. D. (2008). Convergent LMI relaxations for robust analysis of uncertain linear systems using lifted polynomial parameter-dependent Lyapunov functions. Sys. Contr. Lett. 57(8), 680-689.

Oliveira, R. C. L. F., \& Peres, P. L. D. (2006). LMI conditions for robust stability analysis based on polynomially parameter-dependent Lyapunov functions. Sys. Contr. Lett. 55(1), 52-61.

Prajna, S., Papachristodoulou, A., Seiler, P., \& Parrilo, P. A. (2004). SOSTOOLS sum of squares optimization toolbox for MATLAB. Users guide. (available online at http://www.cds.caltech.edu/sostools).

Savkin, A. V., \& Petersen, I. R. (1999). Weak robust controllability and observability of uncertain linear systems. IEEE Trans. Automat. Contr. 44(5), 1037-
1041.

Scherer, C. W., \& Hol, C. W. J. (2006). Matrix sumof-squares relaxations for robust semi-definite programs. Mathematical Programming. 107(1-2), 189211.

Siljak, D. D. (1991). Decentralized control of complex systems. Cambridge: Academic Press.

Sojoudi, S., \& Aghdam, A. G. (2007). Characterizing all classes of LTI stabilizing structurally constrained controllers by means of combinatorics. Proc. 46 th IEEE Conf. Decision and Contr. New Orleans, LO (pp. 4415-4420).

Ugrinovskii, V. A. (2005). Robust controllability of linear stochastic uncertain systems. Automatica. 41(5), 807-813.

Wang, S. H., \& Davison, E. J. (1973). On the stabilization of decentralized control systems. IEEE Trans. Automat. Contr. 18(5), 473-478. 